\title{
Positive Periodic Solution for the Generalized Neutral Differential Equation with Multiple Delays and Impulse
}

\author{
Zhenguo Luo, ${ }^{1,2}$ Liping Luo, ${ }^{2}$ and Yunhui Zeng ${ }^{2}$ \\ ${ }^{1}$ Department of Mathematics, National University of Defense Technology, Changsha 410073, China \\ ${ }^{2}$ Department of Mathematics, Hengyang Normal University, Hengyang 421008, China
}

Correspondence should be addressed to Zhenguo Luo; robert186@163.com

Received 29 June 2013; Revised 8 November 2013; Accepted 11 November 2013; Published 27 February 2014

Academic Editor: Chein-Shan Liu

Copyright (c) 2014 Zhenguo Luo et al. This is an open access article distributed under the Creative Commons Attribution License, which permits unrestricted use, distribution, and reproduction in any medium, provided the original work is properly cited.

By using a fixed point theorem of strict-set-contraction, which is different from Gaines and Mawhin's continuation theorem and abstract continuation theory for $k$-set contraction, we established some new criteria for the existence of positive periodic solution of the following generalized neutral delay functional differential equation with impulse: $x^{\prime}(t)=x(t)[a(t)-f(t, x(t), x(t-$ $\left.\left.\left.\tau_{1}(t, x(t))\right), \ldots, x\left(t-\tau_{n}(t, x(t))\right), x^{\prime}\left(t-\gamma_{1}(t, x(t))\right), \ldots, x^{\prime}\left(t-\gamma_{m}(t, x(t))\right)\right)\right], t \neq t_{k}, k \in Z_{+} ; x\left(t_{k}^{+}\right)=x\left(t_{k}^{-}\right)+\theta_{k}\left(x\left(t_{k}\right)\right), k \in Z_{+}$. As applications of our results, we also give some applications to several Lotka-Volterra models and new results are obtained.

\section{Introduction}

Many systems in physics, chemistry, biology, and information science have impulsive dynamical behavior due to abrupt jumps at certain instants during the evolving processes. This complex dynamical behavior can be modeled by impulsive differential equations. Impulsive differential equations have become more important in recent years in some mathematical models of real processes and phenomena studied in physics, chemical technology, population dynamics, biotechnology, and economics; see [1-8]. There has been a significant development in impulse theory, in recent years, especially in the area of impulsive differential equations with fixed moments; see the monographs [9-11].

In this paper, we consider more general neutral delay functional differential equation with impulse:

$$
\begin{gathered}
x^{\prime}(t)=x(t)\left[a(t)-f\left(t, x(t), x\left(t-\tau_{1}(t, x(t))\right), \ldots,\right.\right. \\
x\left(t-\tau_{n}(t, x(t))\right), \\
x^{\prime}\left(t-\gamma_{1}(t, x(t))\right), \ldots, \\
\left.\left.x^{\prime}\left(t-\gamma_{m}(t, x(t))\right)\right)\right], \\
t \neq t_{k}, k \in Z_{+}, \\
x\left(t_{k}^{+}\right)=x\left(t_{k}^{-}\right)+\theta_{k}\left(x\left(t_{k}\right)\right), \quad k \in Z_{+},
\end{gathered}
$$

where $a \in C\left(R, R^{+}\right), \tau_{i}(t), \gamma_{j}(t) \in C(R, R)(i=1,2, \ldots, n, j=$ $1,2, \ldots, m)$ are $\omega$-periodic functions and $f \in C\left(R^{2+n+m}, R\right)$ is $\omega$-periodic function with respect to its first argument. Moreover, $x\left(t_{k}^{+}\right), x\left(t_{k}^{-}\right)$represents the right, left limit of $x(t)$ at the point $t_{k}$, respectively. In this paper, it is assumed that $x$ is left continuous at $t_{k}$; that is, $x$ changes decreasingly suddenly at times $t_{k} . \theta_{k} \in C\left(R^{+}, R^{+}\right), \omega>0$ is a constant, $R=$ $(-\infty,+\infty)$, and $R^{+}=[0,+\infty), Z_{+}=\{1,2,3, \ldots\}$. We assume that there exists an integer $q>0$ such that $t_{k+q}=t_{k}+\omega$, $\theta_{k+q}=\theta_{k}$, where $0<t_{1}<t_{2}<\cdots<t_{q}<\omega$. For the ecological justification of (1) and the similar types refer to [8, 12-17].

In 1993, Kuang in [12] proposed an open problem (open problem 9.2) to obtain sufficient conditions for the existence of a positive periodic solution of the following equation:

$$
\begin{aligned}
& \frac{d N}{d t}=N(t)[ a(t)-\beta(t) N(t)-b(t) N(t-\tau(t)) \\
&\left.-c(t) N^{\prime}(t-\tau(t))\right] .
\end{aligned}
$$

In [13], Fang and Li studied model (2) and gave an answer to the open problem 9.2 of [12]. But paper [13] required that $b(t) \geq 0, c(t) \geq 0$ and $c_{0}^{\prime}(t)>b(t), \beta(t) \geq 0$ or $c_{0}^{\prime}(t) \leq b(t)$, $\beta(t) \leq 0$ for $t \in[0, \omega]$, where $c_{0}(t)=c(t) /\left(1-\tau^{\prime}(t)\right)$. In [14], 
Yang and Cao studied a general neutral delay model of singlespecies population growth:

$$
\begin{aligned}
\frac{d N}{d t}=N(t)\left[a(t)-\beta(t) N(t)-\sum_{i=1}^{n} b_{i}(t) N\left(t-\tau_{i}(t)\right)\right. \\
\\
\left.\quad-\sum_{i=1}^{n} c_{i}(t) N^{\prime}\left(t-\gamma_{i}(t)\right)\right] .
\end{aligned}
$$

They applied the theory of coincidence degree to obtain verifiable sufficient conditions of the existence of positive periodic solutions of system (3). In [15], Lu considered the following neutral functional differential equation:

$$
\begin{aligned}
\frac{d N}{d t}=N(t)\left[a(t)-\beta(t) N(t)-\sum_{i=1}^{n} b_{i}(t) N\left(t-\tau_{i}(t)\right)\right. \\
\\
\left.\quad-\sum_{j=1}^{m} c_{j}(t) N^{\prime}\left(t-\gamma_{j}(t)\right)\right] .
\end{aligned}
$$

He obtained some sufficient conditions for the existence of positive periodic solutions of model (4) by using the theory of abstract continuous theorem of $k$-set contractive operator and some analysis techniques. In [16], Yang and Cao used the theory of coincidence degree to investigate a complex neutral equation with several state-dependent delays as follows:

$$
\begin{aligned}
\frac{d N}{d t}=N(t)[ & a(t)-\beta(t) N(t)-\sum_{i=1}^{n} b_{i}(t) N\left(t-\tau_{i}(t, N(t))\right) \\
& \left.-\sum_{i=1}^{n} c_{i}(t) N^{\prime}\left(t-\gamma_{i}(t)\right)\right] .
\end{aligned}
$$

They also got some verifiable sufficient conditions of the existence of positive periodic solutions of system (5). In [17], Li and Kuang considered the periodic Lotka-Volterra equation with state-dependent delays:

$$
\begin{aligned}
\frac{d x}{d t}=x(t) & {\left[r(t)-a(t) x(t)+\sum_{i=1}^{n} b_{i}(t) x\left(t-\tau_{i}(t, x(t))\right)\right.} \\
& \left.-\sum_{j=1}^{m} c_{j}(t) x^{\prime}\left(t-\gamma_{j}(t, x(t))\right)\right] .
\end{aligned}
$$

They used the continuation theorem of coincidence degree theory to obtain some sufficient and realistic conditions for the existence of positive periodic solutions of system (6). In
[8], Wang and Dai investigated the following periodic neutral population model with delays and impulse:

$$
\begin{gathered}
\frac{d N}{d t}=N(t)\left[a(t)-e(t) N(t)-\sum_{j=1}^{n} b_{j}(t) N\left(t-\sigma_{j}(t)\right)\right. \\
\left.-\sum_{i=1}^{m} c_{i}(t) N^{\prime}\left(t-\tau_{i}(t)\right)\right], \quad t \neq t_{k}, \\
N\left(t^{+}\right)=\left(1+\theta_{k}\right) N\left(t_{k}\right), \quad k=1,2, \ldots
\end{gathered}
$$

They obtained some sufficient conditions for the existence of positive periodic solutions of model (7) by using the theory of abstract continuous theorem of $k$-set contractive operator and some analysis techniques.

The main purpose of this paper is to establish new criteria to guarantee the existence of positive periodic solutions of the system (1) by using a fixed point theorem of strict-setcontraction [18-20].

For convenience, we introduce the notation

$$
\begin{gathered}
h^{M}=\max _{t \in[0, \omega]}\{h(t)\}, \quad h^{L}=\min _{t \in[0, \omega]}\{h(t)\}, \\
\delta=\lim _{u \rightarrow 0} \sup _{t \leq t_{k} \leq t+\omega} \frac{\theta_{k}(u)}{u}, \quad \sigma=e^{-\int_{0}^{\omega} a(t) d t,} \\
B_{1}=\int_{0}^{\omega}\left[\sigma \beta(t)+\sigma \sum_{i=1}^{n} b_{i}(t)-\sum_{j=1}^{m} c_{j}(t)\right] d t, \\
B_{2}=\int_{0}^{\omega}\left[\beta(t)+\sum_{i=1}^{n} b_{i}(t)+\sum_{j=1}^{m} c_{j}(t)\right] d t,
\end{gathered}
$$

where $h(t)$ is a continuous $\omega$-periodic function.

Throughout this paper, we assume the following.

$\left(A_{1}\right) a, \tau_{i}, \gamma_{j} \in C(R, R)$ are $\omega$-periodic functions. In addition, $a(t) \geq 0, t \in[0, \omega]$, and $\sigma=e^{-\int_{0}^{\omega} a(\xi) d \xi}<1$.

$\left(A_{2}\right) f \in C\left(R^{2+n+m}, R\right)$ is $\omega$-periodic function with $f(t+$ $\omega, \cdot)=f(t, \cdot), f(t, 0, \ldots, 0)=0$.

$\left(A_{3}\right)$ There exist $\omega$-periodic functions $\beta(t), b_{i}(t) \in C(R$, $\left.R^{+}\right), c_{j}(t) \in C^{1}\left(R, R^{+}\right)$, such that

$$
\begin{gathered}
\sigma \beta(t)+\sigma \sum_{i=1}^{n} b_{i}(t)-\sum_{j=1}^{m} c_{j}(t)>0, \\
\mid f\left(t, x_{0}, x_{1}, \ldots, x_{n}, y_{1}, \ldots, y_{m}\right) \\
-f\left(t, x_{0}^{*}, x_{1}^{*}, \ldots, x_{n}^{*}, y_{1}^{*}, \ldots, y_{m}^{*}\right) \mid \\
\leq \beta(t)\left|x_{0}-x_{0}^{*}\right| \\
\quad+\sum_{i=1}^{n} b_{i}(t)\left|x_{i}-x_{i}^{*}\right|+\sum_{j=1}^{m} c_{j}(t)\left|y_{j}-y_{j}^{*}\right|,
\end{gathered}
$$




$$
\begin{aligned}
& f\left(t, x_{0}, x_{1}, \ldots, x_{n}, y_{1}, \ldots, y_{m}\right) \\
& \quad \geq \beta(t) x_{0}+\sum_{i=1}^{n} b_{i}(t) x_{i}-\sum_{j=1}^{m} c_{j}(t) y_{j},
\end{aligned}
$$

where $t \in[0, \omega], \sigma\left|y_{j}\right| \leq x_{i}, \sigma\left|y_{j}^{*}\right| \leq x_{i}^{*}$, and $i=1,2, \ldots, n$, $j=1,2, \ldots, m$.

$\left(A_{4}\right)$ We assume that $\left(1+a^{L}\right) \sigma^{2} B_{1} /(1-\sigma) \geq$ $\max _{t \in[0, \omega]}\left\{\beta(t)+\sum_{i=1}^{n} b_{i}(t)+\sum_{j=1}^{m} c_{j}(t)\right\}$.

$\left(A_{5}\right)$ We assume that $\left(a^{M}-1\right) B_{2} / \sigma(1-\sigma) \leq$ $\min _{t \in[0, \omega]}\left\{\sigma \beta(t)+\sigma \sum_{i=1}^{n} b_{i}(t)-\sum_{j=1}^{m} c_{j}(t)\right\}$.

$\left(A_{6}\right)$ We assume that $\left((1-\sigma) / \sigma^{2} B_{1}\right) \sum_{j=1}^{m} c_{j}^{M}<1$.

The paper is organized as follows. In the next section, we give some definitions and lemmas to prove the main results of this paper. In Section 3, we established some criteria to guarantee the existence of at least one positive periodic solution of system (1) by using a fixed point theorem of strictset-contraction. As applications in Section 4, we study some particular cases of system (1) which have been investigated extensively in the references mentioned previously.

\section{Preliminaries}

In order to obtain the existence of a periodic solution of system (1), we first introduce some definitions and lemmas.

Definition 1 (see [17]). A function $x: R \rightarrow(0,+\infty)$ is said to be a positive solution of (1), if the following conditions are satisfied:

(a) $x(t)$ is absolutely continuous on each $\left(t_{k}, t_{k+1}\right)$;

(b) for each $k \in Z_{+}, x\left(t_{k}^{+}\right)$and $x\left(t_{k}^{-}\right)$exist, and $x\left(t_{k}^{-}\right)=$ $x\left(t_{k}\right)$

(c) $x(t)$ satisfies the first equation of (1) for almost everywhere in $R$ and $x\left(t_{k}\right)$ satisfies the second equation of (1) at impulsive point $t_{k}, k \in Z_{+}$.

Definition 2 (see [18]). Let $X$ be a real Banach space and $E$ a closed, nonempty subset of $X . E$ is a cone provided that

(i) $\alpha x+\beta y \in E$ for all $x, y \in E$ and all $\alpha, \beta \geq 0$;

(ii) $x,-x \in E$ imply $x=0$.

Definition 3 (see [18]). Let $A$ be a bounded subset in $X$. Define $\alpha_{X}(A)=\inf \{\delta>0$ : there is a finite number of subsets $A_{i} \subset A$ such that $A=\bigcup_{i} A_{i}$ and $\left.\operatorname{diam}\left(A_{i}\right) \leq \delta\right\}$, where $\operatorname{diam}\left(A_{i}\right)$ denotes the diameter of the set $A_{i}$; obviously, $0 \leq \alpha_{X}(A)<\infty$. So $\alpha_{X}(A)$ is called the Kuratowski measure of noncompactness of $X$.

Definition 4 (see [18]). Let $X, Y$ be two Banach spaces and $D \subset X$; a continuous and bounded map $T: D \rightarrow Y$ is called $k$-set contractive if for any bounded set $S \subset D$ we have

$$
\alpha_{Y}(T(S)) \leq k \alpha_{X}(S)
$$

$T$ is called strict-set-contractive if it is $k$-set contractive for some $0 \leq k<1$.

Definition 5 (see [19]). The set $F \in P C_{\omega}$ is said to be quasiequicontinuous in $[0, \omega]$, if for any $\epsilon>0$, there exists $\delta>0$ such that if $x \in F, k \in N^{+}, t_{1}, t_{2} \in\left(t_{k-1}, t_{k}\right) \cap[0, \omega]$, and $\left|t_{1}-t_{2}\right|<\delta$, then $\left|x\left(t_{1}\right)-x\left(t_{2}\right)\right|<\epsilon$.

Lemma 6 (see [19]). The set $F \subset P C_{\omega}$ is relatively compact if and only if

(1) $F$ is bounded, that is, $\|x\| \leq M$, for each $x \in F$, and some $M>0$

(2) $F$ is quasiequicontinuous in $[0, \omega]$.

Lemma 7. $x(t)$ is an $\omega$-periodic solution of (1) is equivalent to $x(t)$ is an $\omega$-periodic solution of the following equation:

$$
\begin{gathered}
x(t)=\int_{t}^{t+\omega}[G(t, s) x(s) f(t, x(s), \\
x\left(s-\tau_{1}(s, x(s))\right), \ldots, \\
x\left(s-\tau_{n}(s, x(s))\right), \\
x^{\prime}\left(s-\gamma_{1}(s, x(s))\right), \ldots, \\
\left.\left.x^{\prime}\left(s-\gamma_{m}(s, x(s))\right)\right)\right] d s \\
+\sum_{t \leq t_{k}<t+\omega} G\left(t, t_{k}\right) \theta_{k}\left(x\left(t_{k}\right)\right),
\end{gathered}
$$

where

$$
G(t, s)=\frac{e^{-\int_{t}^{s} a(\xi) d \xi}}{1-e^{-\int_{0}^{\omega} a(\xi) d \xi}}, \quad s \in[t, t+\omega]
$$

Proof. Assume that $x(t) \in X$ is a periodic solution of (1). Then, we have

$$
\begin{aligned}
\frac{d}{d t}\left[x(t) e^{-\int_{0}^{t} a(\xi) d \xi}\right] & \\
=e^{-\int_{0}^{t} a(\xi) d \xi} x(t) f( & t, x(t), x\left(t-\tau_{1}(t, x(t))\right), \ldots, \\
& x\left(t-\tau_{n}(t, x(t))\right), \\
& x^{\prime}\left(t-\gamma_{1}(t, x(t))\right), \ldots, \\
& \left.x^{\prime}\left(t-\gamma_{m}(t, x(t))\right)\right), \quad t \neq t_{k} .
\end{aligned}
$$

Integrating the above equation over $[t, t+\omega]$, we can have

$$
\begin{gathered}
\left.x(s) e^{-\int_{0}^{s} a(\xi) d \xi}\right|_{t} ^{t_{m_{1}}+n \omega}+\left.x(s) e^{-\int_{0}^{s} a(\xi) d \xi}\right|_{t_{m_{1}}+n \omega} ^{t_{m_{2}}+n \omega} \\
+\cdots+\left.x(s) e^{-\int_{0}^{s} a(\xi) d \xi}\right|_{t_{m_{q}}+n \omega} ^{t+\omega}
\end{gathered}
$$




$$
\begin{gathered}
=\int_{t}^{t+\omega}\left[x(s) e^{-\int_{0}^{s} a(\xi) d \xi}\right. \\
\times f\left(t, x(s), x\left(s-\tau_{1}(s, x(s))\right), \ldots,\right. \\
\quad x\left(s-\tau_{n}(s, x(s))\right), \\
x^{\prime}\left(s-\gamma_{1}(s, x(s))\right), \ldots, \\
\left.\left.x^{\prime}\left(s-\gamma_{m}(s, x(s))\right)\right)\right] d s
\end{gathered}
$$

where $t_{m_{k}}+n \omega \in(t, t+\omega), m_{k} \in\{1,2, \ldots, q\}, k=1,2, \ldots, q$, $n \in Z_{+}$. Therefore,

$$
\begin{gathered}
x(t) e^{-\int_{0}^{t} a(\xi) d \xi}\left[1-e^{-\int_{t}^{t+\omega} a(\xi) d \xi}\right] \\
+\sum_{t \leq t_{k}<t+\omega} \Delta x\left(t_{m_{k}}\right) e^{-\int_{0}^{t_{m}+n \omega} a(\xi) d \xi} \\
=\int_{t}^{t+\omega}\left[x(s) e^{-\int_{0}^{s} a(\xi) d \xi}\right. \\
\times f\left(t, x(s), x\left(s-\tau_{1}(s, x(s))\right), \ldots,\right. \\
x\left(s-\tau_{n}(s, x(s))\right), \\
x^{\prime}\left(s-\gamma_{1}(s, x(s))\right), \ldots, \\
\left.\left.x^{\prime}\left(s-\gamma_{m}(s, x(s))\right)\right)\right] d s,
\end{gathered}
$$

which can be transformed into

$$
\begin{gathered}
x(t)=\int_{t}^{t+\omega}[G(t, s) x(s) \\
\times f\left(t, x(s), x\left(s-\tau_{1}(s, x(s))\right), \ldots,\right. \\
x\left(s-\tau_{n}(s, x(s))\right), \\
x^{\prime}\left(s-\gamma_{1}(s, x(s))\right), \ldots, \\
\left.\left.x^{\prime}\left(s-\gamma_{m}(s, x(s))\right)\right)\right] d s \\
+\sum_{t \leq t_{k}<t+\omega} G\left(t, t_{k}\right) \theta_{k}\left(x\left(t_{k}\right)\right) .
\end{gathered}
$$

Thus, $x$ is a periodic solution for (11).

If $x(t) \in E$ is a periodic solution of (11), for any $t=t_{k}$, from (11) we have

$$
\begin{aligned}
& x^{\prime}(t)=\frac{d}{d t}\left\{\int_{t}^{t+\omega}[\right. G(t, s) x(s) \\
& \times f\left(t, x(s), x\left(s-\tau_{1}(s, x(s))\right), \ldots,\right. \\
& x\left(s-\tau_{n}(s, x(s))\right), \\
& x^{\prime}\left(s-\gamma_{1}(s, x(s))\right), \ldots, \\
&\left.\left.\left.x^{\prime}\left(s-\gamma_{m}(s, x(s))\right)\right)\right] d s\right\}
\end{aligned}
$$

$$
\begin{gathered}
=[G(t, t+\omega) x(t+\omega) \\
\times f(t+\omega, x(t+\omega), \\
x\left(t+\omega-\tau_{1}(t+\omega, x(t+\omega))\right), \ldots, \\
x\left(t+\omega-\tau_{n}(t+\omega, x(t+\omega))\right), \\
x^{\prime}\left(t+\omega-\gamma_{1}(t+\omega, x(t+\omega))\right), \ldots, \\
\left.x^{\prime}\left(t+\omega-\gamma_{m}(t+\omega, x(t+\omega))\right)\right) \\
-G(t, t) x(t) f\left(t, x(t), x\left(t-\tau_{1}(t, x(t))\right), \ldots,\right. \\
x\left(t-\tau_{n}(t, x(t))\right), \\
x^{\prime}\left(t-\gamma_{1}(t, x(t))\right), \ldots, \\
\left.\left.x^{\prime}\left(t-\gamma_{m}(t, x(t))\right)\right)\right]+a(t) x(t) \\
x\left(t, x(t), x\left(t-\tau_{1}(t, x(t))\right), \ldots,\right. \\
x\left(t-\tau_{n}(t, x(t))\right), \\
x^{\prime}\left(t-\gamma_{1}(t, x(t))\right), \ldots, \\
\left.\left.x^{\prime}\left(t-\gamma_{m}(t, x(t))\right)\right)\right] . \\
=x(t)[a(t)-f(t), \ldots(t), \ldots
\end{gathered}
$$

For any $t=t_{j}, j \in Z_{+}$, we have from (11) that

$$
\begin{gathered}
x\left(t_{j}^{+}\right)-x\left(t_{j}\right)=\int_{t_{j}}^{t_{j}+\omega}\left[G\left(t_{j}^{+}, s\right)-G\left(t_{j}, s\right)\right] x(s) \\
\times f\left(t, x(s), x\left(s-\tau_{1}(s, x(s))\right), \ldots,\right. \\
x\left(s-\tau_{n}(s, x(s))\right), \\
x^{\prime}\left(s-\gamma_{1}(s, x(s))\right), \ldots, \\
\left.x^{\prime}\left(s-\gamma_{m}(s, x(s))\right)\right) d s \\
+\sum_{t_{j}^{+} \leq t_{k}<t_{j}+\omega} G\left(t_{j}^{+}, t_{k}\right) \theta_{k}\left(x\left(t_{k}\right)\right) \\
-\sum_{t_{j} \leq t_{k}<t_{j}+\omega} G\left(t_{j}, t_{k}\right) \theta_{k}\left(x\left(t_{k}\right)\right) \\
=\theta_{k}\left(x\left(t_{k}\right)\right) .
\end{gathered}
$$

Hence $x(t)$ is a positive $\omega$-periodic solution of (1). Thus we complete the proof of Lemma 7.

Lemma 8 (see [18-20]). Let $E$ be a cone of the real Banach space $X$ and $E_{r, R}=\{x \in E: r \leq\|x\| \leq R\}$ with $0<r<R$. Assume that $A: E_{r, R} \rightarrow E$ is strict-set-contractive such that one of the following two conditions is satisfied:

(a) $A x \not x, \forall x \in E,\|x\|=r$ and $A x \nsupseteq x, \forall x \in E$, $\|x\|=R$ 
(b) $A x \geq x, \forall x \in E,\|x\|=r$ and $A x \npreceq x, \forall x \in E,\|x\|=$ $R$.

Then $A$ has at least one fixed point in $E_{r, R}$.

In order to apply Lemma 8 to system (1), we set

$$
\begin{gathered}
P C(R)=\left\{x: R \longrightarrow R \mid x \in C\left(\left(t_{k}, t_{k+1}\right), R\right),\right. \\
\left.\exists x\left(t_{k}^{-}\right)=x\left(t_{k}\right), x\left(t_{k}^{+}\right), k \in Z_{+}, t \in R\right\}, \\
P C^{1}(R)=\left\{x: R \longrightarrow R \mid x \in C^{1}\left(\left(t_{k}, t_{k+1}\right), R\right),\right. \\
\left.\exists x^{\prime}\left(t_{k}^{-}\right)=x^{\prime}\left(t_{k}\right), x\left(t_{k}^{+}\right), k \in Z_{+}, t \in R\right\} .
\end{gathered}
$$

Define

$$
X=\{x: x \in P C(R) \mid x(t+\omega)=x(t)\}
$$

with the norm defined by $\|x\|=\max _{t \in[0, \omega]}\{|x(t)|\}$ and

$$
Y=\left\{x: x \in P C^{1}(R) \mid x(t+\omega)=x(t), t \in R\right\}
$$

with the norm defined by $\|x\|_{1}=\max \left\{\|x\|,\left\|x^{\prime}\right\|\right\}$. Then $X$ and $Y$ are both Banach spaces. Define the cone $E$ in $Y$ by

$$
E=\left\{x: x \in P C^{1}(R) \mid x(t) \geq \sigma\|x\|_{1}, t \in[0, \omega]\right\} .
$$

Let the map $\phi$ be defined by

$$
\begin{gathered}
(\phi x)(t)=\int_{t}^{t+\omega}[G(t, s) x(s) \\
\times f\left(s, x(s), x\left(s-\tau_{1}(s, x(s))\right), \ldots,\right. \\
x\left(s-\tau_{n}(s, x(s))\right), \\
\quad x^{\prime}\left(s-\gamma_{1}(s, x(s))\right), \ldots \\
\left.\left.\quad x^{\prime}\left(s-\gamma_{m}(s, x(s))\right)\right)\right] d s \\
+\sum_{t \leq t_{k}<t+\omega} G\left(t, t_{k}\right) \theta_{k}\left(x\left(t_{k}\right)\right)
\end{gathered}
$$

where $x \in E, t \in R$, and $G(t, s)$ is defined by (12). It is obvious to see that $G(t+\omega, s+\omega)=G(t, s), \partial G(t, s) / \partial t=a(t) G(t, s)$, $G(t, t+\omega)-G(t, t)=-1$, and

$$
\frac{\sigma}{1-\sigma} \leq G(t, s) \leq \frac{1}{1-\sigma}, \quad s \in[t, t+\omega] .
$$

In what follows, we will give some lemmas concerning $E$ and $\phi$ defined by (22) and (23), respectively.

Lemma 9. Assume that $\left(A_{1}\right)-\left(A_{4}\right)$ hold.

(i) If $a^{M} \leq 1$, then $\phi: E \rightarrow E$ is well defined.

(ii) If $\left(A_{5}\right)$ holds and $a^{M}>1$, then $\phi: E \rightarrow E$ is well defined.
Proof. For any $x \in E$, it is clear that $\phi x \in P C^{1}(R)$. From (23), for $t \in[0, \omega]$, we have

$$
\begin{aligned}
& (\phi x)(t+\omega) \\
& =\int_{t+\omega}^{t+2 \omega}[G(t+\omega, s) x(s) \\
& \times f\left(s, x(s), x\left(s-\tau_{1}(s, x(s))\right), \ldots,\right. \\
& x\left(s-\tau_{n}(s, x(s))\right), \\
& x^{\prime}\left(s-\gamma_{1}(s, x(s))\right), \ldots, \\
& \left.\left.x^{\prime}\left(s-\gamma_{m}(s, x(s))\right)\right)\right] d s \\
& +\sum_{t+\omega \leq t_{k}<t+2 \omega} G\left(t+\omega, t_{k}\right) \theta_{k}\left(x\left(t_{k}\right)\right) \\
& =\int_{t}^{t+\omega}[G(t+\omega, u+\omega) x(u+\omega) \\
& \times f(u+\omega, x(u+\omega), \\
& x\left(u+\omega-\tau_{1}(u+\omega, x(u+\omega))\right), \ldots, \\
& x\left(u+\omega-\tau_{n}(u+\omega, x(u+\omega))\right), \\
& x^{\prime}\left(u+\omega-\gamma_{1}(u+\omega, x(u+\omega))\right), \ldots, \\
& \left.\left.x^{\prime}\left(u+\omega-\gamma_{m}(u+\omega, x(u+\omega))\right)\right)\right] d u \\
& +\sum_{t \leq t_{k}<t+\omega} G\left(t, t_{k}\right) \theta_{k}\left(x\left(t_{k}\right)\right) \\
& =\int_{t}^{t+\omega}[G(t, s) x(s) \\
& \times f\left(s, x(s), x\left(s-\tau_{1}(s, x(s))\right), \ldots,\right. \\
& x\left(s-\tau_{n}(s, x(s))\right), \\
& x^{\prime}\left(s-\gamma_{1}(s, x(s))\right), \ldots, \\
& \left.\left.x^{\prime}\left(s-\gamma_{m}(s, x(s))\right)\right)\right] d s \\
& +\sum_{t \leq t_{k}<t+\omega} G\left(t, t_{k}\right) \theta_{k}\left(x\left(t_{k}\right)\right)=(\phi x)(t) .
\end{aligned}
$$

That is, $(\phi x)(t+\omega)=(\phi x)(t), t \in[0, \omega]$. So $\phi x \in Y$. In view of $\left(A_{3}\right)$, for $x \in E, t \in[0, \omega]$, we have

$$
\begin{aligned}
& f\left(t, x(t), x\left(t-\tau_{1}(t, x(t))\right), \ldots,\right. \\
& \quad x\left(t-\tau_{n}(t, x(t))\right), x^{\prime}\left(t-\gamma_{1}(t, x(t))\right), \ldots, \\
& \left.\quad x^{\prime}\left(t-\gamma_{m}(t, x(t))\right)\right)
\end{aligned}
$$




$$
\begin{aligned}
& \geq \beta(t) x(t)+\sum_{i=1}^{n} b_{i}(t) x\left(t-\tau_{i}(t, x(t))\right) \\
& \quad-\sum_{j=1}^{n} c_{j}(t) x^{\prime}\left(t-\gamma_{j}(t, x(t))\right) \\
& \geq \sigma \beta(t)\left\|x^{\prime}\right\|+\sum_{i=1}^{n} b_{i}(t) \sigma\left\|x^{\prime}\right\|-\sum_{j=1}^{n} c_{j}(t)\left\|x^{\prime}\right\| \\
& =\left\|x^{\prime}\right\|\left[\sigma \beta(t)+\sum_{i=1}^{n} b_{i}(t) \sigma-\sum_{j=1}^{n} c_{j}(t)\right]>0,
\end{aligned}
$$

$$
\begin{aligned}
& f\left(t, x(t), x\left(t-\tau_{1}(t, x(t))\right), \ldots,\right. \\
& x\left(t-\tau_{n}(t, x(t))\right), x^{\prime}\left(t-\gamma_{1}(t, x(t))\right), \ldots, \\
& \left.x^{\prime}\left(t-\gamma_{m}(t, x(t))\right)\right) \\
& \leq \mid f\left(t, x(t), x\left(t-\tau_{1}(t, x(t))\right), \ldots,\right. \\
& x\left(t-\tau_{n}(t, x(t))\right), \\
& x^{\prime}\left(t-\gamma_{1}(t, x(t))\right), \ldots, \\
& \left.x^{\prime}\left(t-\gamma_{m}(t, x(t))\right)\right)-f(t, 0, \ldots, 0) \mid \\
& \leq \beta(t) x(t)+\sum_{i=1}^{n} b_{i}(t) x\left(t-\tau_{i}(t, x(t))\right) \\
& +\sum_{j=1}^{n} c_{j}(t) x^{\prime}\left(t-\gamma_{j}(t, x(t))\right) .
\end{aligned}
$$

Therefore, for $x \in E, t \in[0, \omega]$, we find

$$
\begin{gathered}
\|\phi x\|=\max _{t \in[0, \omega]}\{|\phi x(t)|\} \\
=\max _{t \in[0, \omega]}\left\{\int_{t}^{t+\omega}[G(t, s) x(s)\right. \\
\times f(s, x(s), \\
x\left(s-\tau_{1}(s, x(s))\right), \ldots, \\
x\left(s-\tau_{n}(s, x(s))\right), \\
x^{\prime}\left(s-\gamma_{1}(s, x(s))\right), \ldots, \\
\left.\left.x^{\prime}\left(s-\gamma_{m}(s, x(s))\right)\right)\right] d s \\
\left.+\sum_{t \leq t_{k}<t+\omega} G\left(t, t_{k}\right) \theta_{k}\left(x\left(t_{k}\right)\right)\right\}
\end{gathered}
$$

$$
\begin{gathered}
\leq \frac{1}{1-\sigma}\left\{\int_{0}^{\omega}[x(s) f(s, x(s),\right. \\
x\left(s-\tau_{1}(s, x(s))\right), \ldots, \\
x\left(s-\tau_{n}(s, x(s))\right), \\
x^{\prime}\left(s-\gamma_{1}(s, x(s))\right), \ldots, \\
\left.\left.x^{\prime}\left(s-\gamma_{m}(s, x(s))\right)\right)\right] d s \\
\left.+\sum_{t \leq t_{k}<t+\omega} \theta_{k}\left(x\left(t_{k}\right)\right)\right\} .
\end{gathered}
$$

Furthermore, for $x \in E, t \in[0, \omega]$, we have $(\phi x)(t)$

$$
\begin{aligned}
& \geq \frac{\sigma}{1-\sigma}\left\{\int_{t}^{t+\omega}[x(s) f(s, x(s),\right. \\
& x\left(s-\tau_{1}(s, x(s))\right), \ldots, \\
& x\left(s-\tau_{n}(s, x(s))\right), \\
& x^{\prime}\left(s-\gamma_{1}(s, x(s))\right), \ldots, \\
& \left.\left.x^{\prime}\left(s-\gamma_{m}(s, x(s))\right)\right)\right] d s \\
& \left.+\sum_{t \leq t_{k}<t+\omega} \theta_{k}\left(x\left(t_{k}\right)\right)\right\} \\
& =\frac{\sigma}{1-\sigma}\left\{\int_{0}^{\omega}[x(s) f(s, x(s),\right. \\
& x\left(s-\tau_{1}(s, x(s))\right), \ldots, \\
& x\left(s-\tau_{n}(s, x(s))\right), \\
& x^{\prime}\left(s-\gamma_{1}(s, x(s))\right), \ldots \text {, } \\
& \left.\left.x^{\prime}\left(s-\gamma_{m}(s, x(s))\right)\right)\right] d s \\
& \left.+\sum_{t \leq t_{k}<t+\omega} \theta_{k}\left(x\left(t_{k}\right)\right)\right\} \geq \sigma\|\phi x\| .
\end{aligned}
$$

Now, we show that $(\phi x)(t) \geq \sigma\|\phi x\|, t \in[0, \omega]$. On the other hand, from (23), we obtain

$$
\begin{aligned}
&(\phi x)^{\prime}(t)=G(t, t+\omega) x(t+\omega) \\
& \times f(t+\omega, x(t+\omega), \\
& x\left(t+\omega-\tau_{1}(t+\omega, x(t+\omega))\right), \ldots, \\
& x\left(t+\omega-\tau_{n}(t+\omega, x(t+\omega))\right),
\end{aligned}
$$




$$
\begin{gathered}
x^{\prime}\left(t+\omega-\gamma_{1}(t+\omega, x(t+\omega))\right), \ldots, \\
\left.x^{\prime}\left(t+\omega-\gamma_{m}(t+\omega, x(t+\omega))\right)\right) \\
-G(t, t) x(t) f\left(t, x(t), x\left(t-\tau_{1}(t, x(t))\right), \ldots,\right. \\
x\left(t-\tau_{n}(t, x(t))\right), \\
x^{\prime}\left(t-\gamma_{1}(t, x(t))\right), \ldots, \\
\left.x^{\prime}\left(t-\gamma_{m}(t, x(t))\right)\right) \\
+a(t)(\phi x)(t) \quad \\
=a(t)(\phi x)(t) \quad \\
-x(t) f(t, x(t), \\
x\left(t-\tau_{1}(t, x(t))\right), \ldots, \\
x\left(t-\tau_{n}(t, x(t))\right), \\
x^{\prime}\left(t-\gamma_{1}(t, x(t))\right), \ldots, \\
\left.x^{\prime}\left(t-\gamma_{m}(t, x(t))\right)\right) .
\end{gathered}
$$

It follows from (29) and (30) that if $(\phi x)^{\prime}(t) \geq 0$, then

$$
(\phi x)^{\prime}(t) \leq a(t)(\phi x)(t) \leq a^{M}(\phi x)(t) \leq(\phi x)(t) .
$$

On the other hand, from $(30)$ and $\left(A_{4}\right)$, if $(\phi x)^{\prime}(t)<0$, then

$$
\begin{aligned}
-(\phi x)^{\prime}(t)=-a(t)(\phi x)(t) & \\
& +x(t) f(t, x(t), \\
x\left(t-\tau_{1}(t, x(t))\right), \ldots, & x\left(t-\tau_{n}(t, x(t))\right), \\
& x^{\prime}\left(t-\gamma_{1}(t, x(t))\right), \ldots, \\
\left.x^{\prime}\left(t-\gamma_{m}(t, x(t))\right)\right) & \\
\leq & \|x\|_{1}^{2}\left[\beta(t)+\sum_{i=1}^{n} b_{i}(t)+\sum_{j=1}^{n} c_{j}(t)\right]-a^{L}(\phi x)(t) \\
\leq & \left(1+a^{L}\right) \frac{\sigma^{2}}{1-\sigma}\|x\|_{1}^{2} \\
& \times \int_{t}^{t+\omega}\left[\beta(s)+\sum_{i=1}^{n} b_{i}(s)-\sum_{j=1}^{n} c_{j}(s)\right] d s \\
& -a^{L}(\phi x)(t)
\end{aligned}
$$

$$
\begin{aligned}
& =\left(1+a^{L}\right) \int_{t}^{t+\omega} \frac{\sigma}{1-\sigma} \sigma\|x\|_{1} \\
& \times\left[\beta(s)\|x\|_{1}+\sum_{i=1}^{n} b_{i}(s)\|x\|_{1}\right. \\
& \left.-\sum_{j=1}^{n} c_{j}(s)\|x\|_{1}\right] d s \\
& -a^{L}(\phi x)(t) \\
& \leq\left(1+a^{L}\right) \int_{t}^{t+\omega} G(t, s) x(s) \\
& \times\left[\beta(t) x(s)+\sum_{i=1}^{n} b_{i}(t) x\left(s-\tau_{i}(s)\right)\right. \\
& \left.-\sum_{j=1}^{n} c_{j}(t) x^{\prime}\left(s-\gamma_{j}(s)\right)\right] d s \\
& -a^{L}(\phi x)(t) \\
& =\left(1+a^{L}\right) \int_{t}^{t+\omega} G(t, s) x(s) \\
& \times f\left(s, x(s), x\left(s-\tau_{1}(s, x(s))\right), \ldots,\right. \\
& x\left(s-\tau_{n}(s, x(s))\right), \\
& x^{\prime}\left(s-\gamma_{1}(s, x(s))\right), \ldots, \\
& \left.x^{\prime}\left(s-\gamma_{m}(s, x(s))\right)\right) d s
\end{aligned}
$$

$$
\begin{aligned}
& -a^{L}(\phi x)(t) \\
= & \left(1+a^{L}\right)(\phi x)(t)-a^{L}(\phi x)(t)=(\phi x)(t) .
\end{aligned}
$$

It follows from (31) and (32) that $\left\|(\phi x)^{\prime}\right\| \leq\|\phi x\|$. So $\|\phi x\|_{1}=$ $\|\phi x\|_{0}$. By (29) we have $(\phi x)(t) \geq \sigma\|\phi x\|_{1}$. Hence, $\phi x \in E$. This completes the proof of (i).

(ii) In view of the proof of (i), we only need to prove that $(\phi x)^{\prime}(t) \geq 0$ implies $(\phi x)^{\prime}(t) \leq(\phi x)(t)$. From (23), (26), $\left(A_{3}\right)$, and $\left(A_{5}\right)$, we have

$$
\begin{aligned}
&(\phi x)^{\prime}(t)= a(t)(\phi x)(t)-x(t) \\
& \times f\left(t, x(t), x\left(t-\tau_{1}(t, x(t))\right), \ldots\right. \\
& x\left(t-\tau_{n}(t, x(t))\right) \\
& x^{\prime}\left(t-\gamma_{1}(t, x(t))\right), \ldots, \\
&\left.x^{\prime}\left(t-\gamma_{m}(t, x(t))\right)\right) \\
& \leq a(t)(\phi x)(t)-\sigma\|x\|_{1}
\end{aligned}
$$




$$
\begin{aligned}
& \times f\left(t, x(t), x\left(t-\tau_{1}(t, x(t))\right), \ldots,\right. \\
& x\left(t-\tau_{n}(t, x(t))\right), \\
& x^{\prime}\left(t-\gamma_{1}(t, x(t))\right), \ldots, \\
& \left.x^{\prime}\left(t-\gamma_{m}(t, x(t))\right)\right) \\
& \leq a^{M}(\phi x)(t)-\sigma\|x\|_{1}^{2} \\
& \times\left[\sigma \beta(t)+\sigma \sum_{i=1}^{n} b_{i}(t)-\sum_{j=1}^{n} c_{j}(t)\right] \\
& \leq a^{M}(\phi x)(t)-\sigma\|x\|_{1}^{2} \frac{a^{M}-1}{\sigma(1-\sigma)} \\
& \times \int_{0}^{\omega}\left[\beta(s)+\sum_{i=1}^{n} b_{i}(s)+\sum_{j=1}^{n} c_{j}(s)\right] d s \\
& =a^{M}(\phi x)(t)-\left(a^{M}-1\right) \\
& \times \int_{t}^{t+\omega} \frac{1}{1-\sigma}\|x\|_{1}\left[\beta(s)\|x\|_{1}+\sum_{i=1}^{n} b_{i}(s)\|x\|_{1}\right. \\
& \left.+\sum_{j=1}^{n} c_{j}(s)\|x\|_{1}\right] d s \\
& \leq a^{M}(\phi x)(t)-\left(a^{M}-1\right) \\
& \times \int_{t}^{t+\omega} G(t, s) x(s) \\
& \times\left[\beta(s) x(s)+\sum_{i=1}^{n} b_{i}(s) x\left(s-\tau_{i}(s, x(s))\right)\right. \\
& \left.+\sum_{j=1}^{n} c_{j}(s) x\left(s-\gamma_{j}(s, x(s))\right)\right] d s \\
& \leq a^{M}(\phi x)(t)-\left(a^{M}-1\right) \\
& \times\left\{\int_{t}^{t+\omega} G(t, s) x(s)\right. \\
& \times f\left(s, x(s), x\left(s-\tau_{1}(s, x(s))\right), \ldots,\right. \\
& x\left(s-\tau_{n}(s, x(s))\right), \\
& x^{\prime}\left(s-\gamma_{1}(s, x(s))\right), \ldots, \\
& \left.x^{\prime}\left(s-\gamma_{m}(s, x(s))\right)\right) d s \\
& \left.+\sum_{t \leq t_{k}<t+\omega} G\left(t, t_{k}\right) \theta_{k}\left(x\left(t_{k}\right)\right)\right\} \\
& =a^{M}(\phi x)(t)-\left(a^{M}-1\right)(\phi x)(t)=(\phi x)(t) \text {. }
\end{aligned}
$$

The proof of (ii) is complete. Thus we complete the proof of Lemma 9.

Lemma 10. Assume that $\left(A_{1}\right)-\left(A_{4}\right)$ hold and $R \sum_{j=1}^{n} c_{j}^{M}<1$.

(i) If $a^{M} \leq 1$, then $\phi: E \bigcap \overline{\Omega_{R}} \rightarrow E$ is strict-set-contractive.

(ii) If $\left(A_{5}\right)$ holds and $a^{M}>1$, then $\phi: E \bigcap \overline{\Omega_{R}} \rightarrow E$ is strict-set-contractive,

where $\Omega_{R}=\left\{x \in Y:|x|_{1}<R\right\}$.

Proof. We only need to prove (i), since the proof of (ii) is similar. It is easy to see that $\phi$ is continuous and bounded. Now we prove that a $\alpha_{Y}(\phi(S)) \leq R \sum_{j=1}^{n} c_{j}^{M} \alpha_{Y}(S)$ for any bounded set $S \in \bar{\Omega}_{R}$. Let $\eta=\alpha_{Y}(S)$; then, for any positive number $\epsilon<R \sum_{j=1}^{n} c_{j}^{M} \eta$, there is a finite family of subsets $\left\{S_{i}\right\}$ satisfying $S=\bigcup_{i} S_{i}$ with $\operatorname{diam}\left(S_{i}\right) \leq \eta+\epsilon$. Therefore,

$$
|x-y|_{1} \leq \eta+\epsilon, \quad \text { for any } x, y \in S_{i} \text {. }
$$

As $S$ and $S_{i}$ are precompact in $X$, it follows that there is a finite family of subsets $\left\{S_{i j}\right\}$ of $S_{i}$ such that $S_{i}=\bigcup_{j} S_{i j}$ and

$$
|x-y|_{0} \leq \epsilon, \quad \text { for any } x, y \in S_{i j}
$$

In addition, for any $x \in S$ and $t \in[0, \omega]$, we have

$$
\begin{aligned}
&(\phi x)(t)=\int_{t}^{t+\omega}[G(t, s) x(s) \\
& \times f\left(s, x(s), x\left(s-\tau_{1}(s, x(s))\right), \ldots,\right. \\
& x\left(s-\tau_{n}(s, x(s))\right), \\
& x^{\prime}\left(s-\gamma_{1}(s, x(s))\right), \ldots,\left.\left.x^{\prime}\left(s-\gamma_{m}(s, x(s))\right)\right)\right] d s \\
&+\sum_{t \leq t_{k}<t+\omega} G\left(t, t_{k}\right) \theta_{k}\left(x\left(t_{k}\right)\right) \frac{R^{2}}{1-\sigma} \int_{0}^{\omega}\left[\beta(s)+\sum_{j=1}^{n} b_{i}(s)+\sum_{j=1}^{n} c_{j}(s)\right] d s \\
&+\frac{1}{1-\sigma} \sum_{t \leq t_{k}<t+\omega} \theta_{k}\left(x\left(t_{k}\right)\right):=\Delta,
\end{aligned}
$$




$$
\begin{gathered}
\left|(\phi x)^{\prime}(t)\right|=\mid a(t)(\phi x)(t)-x(t) \\
\times f\left(t, x(t), x\left(t-\tau_{1}(t, x(t))\right), \ldots,\right. \\
x\left(t-\tau_{n}(t, x(t))\right), \\
x^{\prime}\left(t-\gamma_{1}(t, x(t))\right), \ldots, \\
\left.x^{\prime}\left(t-\gamma_{m}(t, x(t))\right)\right) \mid \\
\leq a^{M} \Delta+R^{2}\left(\beta^{M}+\sum_{i=1}^{n} b_{i}^{M}+\sum_{j=1}^{m} c_{j}^{M}\right) .
\end{gathered}
$$

Hence,

$$
\|(\phi x)\| \leq \Delta,
$$

$$
\left\|(\phi x)^{\prime}\right\| \leq a^{M} \Delta+R^{2}\left(\beta^{M}+\sum_{i=1}^{n} b_{i}^{M}+\sum_{j=1}^{m} c_{j}^{M}\right) .
$$

Applying the Arzela-Ascoli theorem, we know that $\phi(S)$ is precompact in $X$. Then, there is a finite family of subsets $\left\{S_{i j k}\right\}$ of $S_{i j}$ such that $S_{i j}=\bigcup_{k} S_{i j k}$ and

$$
|\phi x-\phi y|_{0} \leq \epsilon, \quad \text { for any } x, y \in S_{i j k} .
$$

From (34)-(39) and $\left(A_{3}\right)$, for any $x, y \in S_{i j k}$, we have

$$
\begin{gathered}
\left\|(\phi x)^{\prime}-(\phi y)^{\prime}\right\| \\
=\max _{t \in[0, \omega]}\{\mid a(t)(\phi x)(t)-a(t)(\phi y)(t)-x(t) \\
\times f\left(t, x(t), x\left(t-\tau_{1}(t, x(t))\right), \ldots,\right. \\
x\left(t-\tau_{n}(t, x(t))\right), \\
x^{\prime}\left(t-\gamma_{1}(t, x(t))\right), \ldots, \\
\left.x^{\prime}\left(t-\gamma_{m}(t, x(t))\right)\right) \\
+y(t) f\left(t, y(t), y\left(t-\tau_{1}(t, y(t))\right), \ldots,\right. \\
y\left(t-\tau_{n}(t, y(t))\right), \\
y^{\prime}\left(t-\gamma_{1}(t, y(t))\right), \ldots, \\
\left.\left.y^{\prime}\left(t-\gamma_{m}(t, y(t))\right)\right) \mid\right\} \\
\leq \max _{t \in[0, \omega]}\{|a(t)((\phi x)(t)-(\phi y)(t))|\}
\end{gathered}
$$

$$
\begin{gathered}
+\max _{t \in[0, \omega]}\left\{\mid x(t) f\left(t, x(t), x\left(t-\tau_{1}(t, x(t))\right), \ldots,\right.\right. \\
x\left(t-\tau_{n}(t, x(t))\right), \\
x^{\prime}\left(t-\gamma_{1}(t, x(t))\right), \ldots, \\
\left.x^{\prime}\left(t-\gamma_{m}(t, x(t))\right)\right) \\
-y(t) f\left(t, y(t), y\left(t-\tau_{1}(t, y(t))\right), \ldots,\right. \\
y\left(t-\tau_{n}(t, y(t))\right), \\
y^{\prime}\left(t-\gamma_{1}(t, y(t, y(t)))\right), \ldots,
\end{gathered}
$$$$
\left.\left.y^{\prime}\left(t-\gamma_{m}(t, y(t))\right)\right) \mid\right\}
$$

$$
\begin{gathered}
\leq a^{M}\|(\phi x)-(\phi y)\| \\
+\max _{t \in[0, \omega]}\left\{\mid x(t)\left[f \left(t, x(t), x\left(t-\tau_{1}(t, x(t))\right), \ldots,\right.\right.\right. \\
x\left(t-\tau_{n}(t, x(t))\right), \\
x^{\prime}\left(t-\gamma_{1}(t, x(t))\right), \ldots, \\
\left.x^{\prime}\left(t-\gamma_{m}(t, x(t))\right)\right) \\
-f\left(t, y(t), y\left(t-\tau_{1}(t, y(t))\right), \ldots,\right. \\
y\left(t-\tau_{n}(t, y(t))\right), \\
y^{\prime}\left(t-\gamma_{1}(t, y(t, y(t)))\right), \ldots, \\
\left.\left.\left.y^{\prime}\left(t-\gamma_{m}(t, y(t))\right)\right)\right] \mid\right\} \\
+\max _{t \in[0, \omega]}\{\mid[x(t)-y(t)] \\
\times f\left(t, y(t), y\left(t-\tau_{1}(t, y(t))\right), \ldots,\right. \\
y\left(t-\tau_{n}(t, y(t))\right), \\
y^{\prime}\left(t-\gamma_{1}(t, y(t, y(t)))\right), \ldots, \\
\left.\left.\left.\left.y^{\prime}\left(t-\gamma_{m}(t, y(t))\right)\right)\right]\right\}\right\} \\
+\max _{t \in[0, \omega]}\{\gamma(t)|x(t)-y(t)| \\
-\sum_{i=1}^{n} b_{i}(t) \mid x\left(t-\tau_{i}(t, x(t))\right) \\
\|(\phi x)-(\phi y)\| \\
\end{gathered}
$$




$$
\begin{gathered}
+\sum_{j=1}^{m} c_{j}(t) \mid x^{\prime}\left(t-\gamma_{j}(t, x(t))\right) \\
\left.-y^{\prime}\left(t-\gamma_{j}(t, y(t))\right) \mid\right\} \\
\max _{t \in[0, \omega]}\left\{\beta(t) y(t)+\sum_{i=1}^{n} b_{i}(t) y\left(t-\tau_{i}(t, y(t))\right)\right. \\
\left.+\sum_{j=1}^{m} c_{j}(t)\left|y^{\prime}\left(t-\gamma_{j}(t, y(t))\right)\right|\right\} \\
\leq a^{M} \epsilon+R \epsilon\left(\beta^{M}+\sum_{i=1}^{n} b_{i}^{M}\right)+R \sum_{j=1}^{m} c_{j}^{M}(\epsilon+\eta) \\
+R \epsilon \sum_{i=1}^{M}\left(\beta^{M}+\sum_{i=1}^{n} b_{i}^{M}+\sum_{j=1}^{m} c_{j}^{M}\right) \\
=\eta\left(\sum_{j=1}^{m} c_{j}^{M} R\right)+\Gamma \epsilon,
\end{gathered}
$$

where

$$
\Gamma:=a^{M}+2 R\left(\beta^{M}+\sum_{i=1}^{n} b_{i}^{M}+\sum_{j=1}^{m} c_{j}^{M}\right) .
$$

From (40) we obtain

$$
\|\phi x-\phi y\|_{1} \leq \eta\left(\sum_{j=1}^{m} c_{j}^{M} R\right)+\Gamma \epsilon, \quad \text { for any } x, y \in S_{i j k} .
$$

As $\epsilon$ is arbitrary small, it follows that

$$
\alpha_{Y}(\phi(S)) \leq\left(R \sum_{j=1}^{n} c_{j}^{M}\right) \alpha_{Y}(S)
$$

Therefore, $\phi$ is strict-set-contractive. The proof of Lemma 10 is complete.

Lemma 11. Assume that $\left(A_{1}\right)-\left(A_{4}\right)$ hold.

(i) If $a^{M} \leq 1$, then $x$ is a positive $\omega$-periodic solution of model (1), where $x$ is a nonzero fixed point of the operator $\phi$ on $E$.

(ii) If $\left(A_{5}\right)$ holds and $a^{M}>1$, then $x$ is a positive $\omega$-periodic solution of model (1), where $x$ is a nonzero fixed point of the operator $\phi$ on $E$.

\section{Main Results}

In this section, we will study the existence of positive $\omega$ periodic solutions of system (1).
Theorem 12. Assume that $\left(A_{1}\right)-\left(A_{4}\right)$, and $\left(A_{6}\right)$ hold.

(i) If $a^{M} \leq 1$, then system (1) has at least one positive $\omega$ periodic solution.

(ii) If $\left(A_{5}\right)$ holds and $a^{M}>1$, then system (1) has at least one positive $\omega$-periodic solution.

Proof. We only need to prove (i), since the proof of (ii) is similar. Let

$$
R=\frac{1-\sigma}{\sigma^{2} B_{1}}, \quad 0<r<\frac{\sigma(1-\sigma)-\xi}{B_{2}} .
$$

Then it is easy to see that $0<r<R$. From Lemmas 9 and 10 , we know that $\phi$ is strict-set-contractive on $E_{r, R}$. By Lemma 11, we see that if there exists $x^{*} \in E$ such that $\phi x^{*}=x^{*}$, then $x^{*}$ is one positive $\omega$-periodic solution of system (1). Now, we will prove that condition (b) of Lemma 8 holds.

First, we prove that $T x \geq x, \forall x \in E,\|x\|_{1}<r$. Otherwise, there exist $x \in E,\|x\|_{1}<r$, such that $T x \geq x$. So $\|x\|>0$ and $\phi x-x \geq 0$, which implies that

$$
(\phi x)(t)-x(t) \geq \sigma\|\phi x-x\|_{1} \geq 0, \quad \text { for any } t \in[0, \omega] .
$$

Moreover, for $t \in[0, \omega]$, we have

$$
\begin{aligned}
(\phi x)(t)=\int_{t}^{t+\omega}[G(t, s) x(s) & \\
& \times f\left(s, x(s), x\left(s-\tau_{1}(s, x(s))\right), \ldots,\right. \\
& x\left(s-\tau_{n}(s, x(s))\right), \\
& x^{\prime}\left(s-\gamma_{1}(s, x(s))\right), \ldots, \\
\left.\left.x^{\prime}\left(s-\gamma_{m}(s, x(s))\right)\right)\right] d s & \\
& +\sum_{t \leq t_{k}<t+\omega} G\left(t, t_{k}\right) \theta_{k}\left(x\left(t_{k}\right)\right) \\
\leq & \frac{r}{1-\sigma}\|x\| \quad \\
& \times\left\{\int_{0}^{\omega}\left[\beta(s)+\sum_{i=1}^{n} b_{i}(s)+\sum_{j=1}^{m} c_{j}(s)\right] d s+\xi\right\} \\
\leq & \frac{B_{2} r+\xi}{1-\sigma}\|x\| \leq \sigma\|x\| .
\end{aligned}
$$

In view of (45) and (46), we obtain

$$
\|x\| \leq\|\phi x\| \leq \sigma\|x\|<\|x\|,
$$

which is a contradiction.

Finally, we prove that $\phi x \nless x, \forall x \in E,\|x\|_{1}=R$. For this case, for the sake of contradiction, suppose that there exist $x \in E,\|x\|_{1}=R$ such that $\phi x \leq x$. Furthermore, for any $t \in[0, \omega]$, we have

$$
x(t)-\phi x(t) \geq \sigma\|x-\phi x\| \geq 0, \quad \text { for any } t \in[0, \omega] .
$$


In addition, for any $t \in[0, \omega]$, we find

$$
\begin{aligned}
(\phi x)(t)=\int_{t}^{t+\omega}[G(t, s) x(s) & \\
& \times f\left(s, x(s), x\left(s-\tau_{1}(s, x(s))\right), \ldots,\right. \\
& x\left(s-\tau_{n}(s, x(s))\right), \\
& x^{\prime}\left(s-\gamma_{1}(s, x(s))\right), \ldots, \\
& \left.\left.+\sum^{\prime}\left(s-\gamma_{m}(s, x(s))\right)\right)\right] d s \\
> & \frac{\sigma^{2}}{1-t_{k}<t+\omega}\|x\|^{2} \\
& \times \int_{0}^{\omega}\left[\begin{array}{l}
\sigma \beta \\
k
\end{array} \theta_{k}\left(x\left(t_{k}\right)\right)\right. \\
= & \frac{\sigma^{2}}{1-\sigma} B \sum_{1} R^{2}=R,
\end{aligned}
$$

which is a contradiction. Therefore, condition (b) of Lemma 8 holds. By Lemma 8 , we see that $\phi$ has at least one nonzero fixed point in $E$. Thus, the system (11) has at least one positive $\omega$-periodic solution. Therefore, it follows from Lemma 7 that system (1) has a positive $\omega$-periodic solution. The proof of Theorem 12 is complete.

\section{Applications}

In this section, we apply the result obtained in the previous section to some periodic population models with impulses which are mentioned in the first section.

First, we consider a general neutral delay model of singlespecies population growth with impulse:

$$
\begin{gathered}
\frac{d N}{d t}=N(t)\left[a(t)-\beta(t) N(t)-\sum_{i=1}^{n} b_{i}(t) N\left(t-\tau_{i}(t)\right)\right. \\
\left.\quad-\sum_{i=1}^{n} c_{i}(t) N^{\prime}\left(t-\gamma_{i}(t)\right)\right], \quad t \neq t_{k}, \quad k \in Z_{+}, \\
N\left(t_{k}^{+}\right)=N\left(t_{k}^{-}\right)+\theta_{k}\left(N\left(t_{k}\right)\right), \quad k \in Z_{+},
\end{gathered}
$$

and we investigate a complex neutral equation with several state-dependent delays and impulse:

$$
\begin{aligned}
\frac{d N}{d t}=N(t)[ & a(t)-\beta(t) N(t) \\
& -\sum_{i=1}^{n} b_{i}(t) N\left(t-\tau_{i}(t, N(t))\right)
\end{aligned}
$$

$$
\begin{gathered}
\left.-\sum_{i=1}^{n} c_{i}(t) N^{\prime}\left(t-\gamma_{i}(t, N(t))\right)\right], \\
t \neq t_{k}, k \in Z_{+}, \\
N\left(t_{k}^{+}\right)=N\left(t_{k}^{-}\right)+\theta_{k}\left(N\left(t_{k}\right)\right), \quad k \in Z_{+} .
\end{gathered}
$$

For convenience, we list several assumptions:

$\left(A_{1}^{*}\right),\left(A_{2}^{*}\right),\left(A_{3}^{*}\right)$, and $\left(A_{4}^{*}\right)$ are the same as $\left(A_{1}\right),\left(A_{4}\right)$, $\left(A_{5}\right)$, and $\left(A_{6}\right)$, respectively;

$\left(A_{5}^{*}\right) \beta(t), b_{i}(t), c_{i}(t) \in C\left(R, R^{+}\right)(i=1,2, \ldots, n)$ are $\omega$-periodic functions and

$$
\sigma \beta(t)+\sigma \sum_{i=1}^{n} b_{i}(t)-\sum_{i=1}^{n} c_{i}(t)>0, \quad t \in[0, \omega] .
$$

Theorem 13. Assume $\left(A_{1}^{*}\right)-\left(A_{3}^{*}\right),\left(A_{5}^{*}\right)$ hold.

(i) If $a^{M} \leq 1$, then systems (50) and (51) have at least one positive $\omega$-periodic solution.

(ii) If $\left(A_{5}^{*}\right)$ holds and $a^{M}>1$, then systems (50) and (51) have at least one positive $\omega$-periodic solution.

Proof. The proof is similar to that of Theorem 12; we omit the details here.

Second, we consider a general neutral delay model of single-species population growth with impulse:

$$
\begin{aligned}
& \frac{d N}{d t}=N(t)[ a(t)-\beta(t) N(t) \\
&-\sum_{i=1}^{n} b_{i}(t) N\left(t-\tau_{i}(t)\right) \\
&\left.-\sum_{j=1}^{m} c_{j}(t) N^{\prime}\left(t-\gamma_{j}(t)\right)\right], \\
& t \neq t_{k}, \quad k \in Z_{+}, \\
& N\left(t_{k}^{+}\right)=N\left(t_{k}^{-}\right)+\theta_{k}\left(N\left(t_{k}\right)\right), \quad k \in Z_{+},
\end{aligned}
$$

and we investigate a periodic Lotka-Volterra equation with state-dependent delays and impulse:

$$
\begin{aligned}
& \frac{d N}{d t}=N(t)[r(t)-a(t) N(t) \\
& -\sum_{i=1}^{n} b_{i}(t) N\left(t-\tau_{i}(t, N(t))\right) \\
& \left.-\sum_{j=1}^{m} c_{j}(t) N^{\prime}\left(t-\gamma_{j}(t, N(t))\right)\right], \\
& t \neq t_{k}, k \in Z_{+}, \\
& N\left(t_{k}^{+}\right)=N\left(t_{k}^{-}\right)+\theta_{k}\left(N\left(t_{k}\right)\right), \quad k \in Z_{+} .
\end{aligned}
$$


For convenience, we list several assumptions:

$\left(H_{1}\right),\left(H_{2}\right),\left(H_{3}\right)$, and $\left(H_{4}\right)$ are the same as $\left(A_{1}\right),\left(A_{4}\right)$, $\left(A_{5}\right)$, and $\left(A_{6}\right)$, respectively;

$\left(H_{5}\right) \beta(t), b_{i}(t), c_{j}(t) \in C\left(R, R^{+}\right)(i=1,2, \ldots, n, j=$ $1,2, \ldots, m)$ are $\omega$-periodic functions and

$$
\sigma \beta(t)+\sigma \sum_{i=1}^{n} b_{i}(t)-\sum_{j=1}^{m} c_{j}(t)>0, \quad t \in[0, \omega]
$$

Then we can obtain the following theorem.

Theorem 14. Assume $\left(H_{1}\right)-\left(H_{4}\right)$ hold.

(i) If $a^{M} \leq 1$, then systems (53) and (54) have at least one positive $\omega$-periodic solution.

(ii) If $\left(H_{5}\right)$ holds and $a^{M}>1$, then systems (53) and (54) have at least one positive $\omega$-periodic solution.

Proof. The proof is similar to that of Theorem 12; we omit the details here.

Remark 15 . We apply the main result obtained in the previous section to study some examples which have some biological implications. A very basic and important ecological problem associated with the study of population is that of the existence of a positive periodic solution which plays the role played by the equilibrium of the autonomous models and means that the species is in an equilibrium state. From Theorems 13 and 14, we see that, under the appropriate conditions, the impulsive perturbations do not affect the existence of periodic solution of systems. Therefore, our result generalizes and improves the corresponding results in [12-17].

\section{Conflict of Interests}

The authors declare that there is no conflict of interests regarding the publication of this paper.

\section{Acknowledgments}

This research is supported by NSF of China (nos. 10971229, 11161015, and 11371367), PSF of China (nos. 2012M512162 and 2013T60934), NSF of Hunan province (nos. 11JJ900, 12JJ9001, and 13JJ4098), the Education Foundation of Hunan province (nos. 12C0541, 12C0361, and 13C084), and the construct program of the key discipline in Hunan province.

\section{References}

[1] Z. G. Luo and L. P. Luo, "Existence and stability of positive periodic solutions for a neutral multispecies logarithmic population model with feedback control and impulse," Abstract and Applied Analysis, vol. 2013, Article ID 741043, 11 pages, 2013.

[2] S. R. Zhang, J. T. Sun, and Y. Zhang, "Stability of impulsive stochastic differential equations in terms of two measures via perturbing Lyapunov functions," Applied Mathematics and Computation, vol. 218, no. 9, pp. 5181-5186, 2012.
[3] Z. G. Luo, B. X. Dai, and Q. Wang, "Existence of positive periodic solutions for a nonautonomous neutral delay $n$-species competitive model with impulses," Nonlinear Analysis: Real World Applications, vol. 11, no. 5, pp. 3955-3967, 2010.

[4] D. W. Lin, X. D. Li, and D. O'Regan, "Stability analysis of generalized impulsive functional differential equations," Mathematical and Computer Modelling, vol. 55, no. 5-6, pp. 1682-1690, 2012.

[5] X. D. Li and X. L. Fu, "On the global exponential stability of impulsive functional differential equations with infinite delays or finite delays," Communications in Nonlinear Science and Numerical Simulation, vol. 19, no. 3, pp. 442-447, 2014.

[6] Q. J. Wu, J. Zhou, and L. Xiang, "Global exponential stability of impulsive differential equations with any time delays," Applied Mathematics Letters, vol. 23, no. 2, pp. 143-147, 2010.

[7] S. M. Afonso, E. M. Bonotto, M. Federson, and L. P. Gimenes, "Stability of functional differential equations with variable impulsive perturbations via generalized ordinary differential equations," Bulletin des Sciences Mathématiques, vol. 137, no. 2, pp. 189-214, 2013.

[8] Q. Wang and B. X. Dai, "Existence of positive periodic solutions for a neutral population model with delays and impulse," Nonlinear Analysis A, vol. 69, no. 11, pp. 3919-3930, 2008.

[9] D. D. Bainov and P. S. Simeonov, Impulsive Differential Equations: Periodic Solutions and Applications, Longman Scientific and Technical, 1993.

[10] A. M. Samoilenko and N. A. Perestyuk, Impulsive Differential Equations, World Scientific Publishing, Singapore, 1995.

[11] S. T. Zavalishchin and A. N. Sesekin, Dynamic Impulse Systems, Kluwer Academic Publishers Group, Dordrecht, The Netherlands, 1997.

[12] Y. Kuang, Delay Differential Equations with Applications in Population Dynamics, Academic Press, Boston, Mass, USA, 1993.

[13] H. Fang and J. B. Li, "On the existence of periodic solutions of a neutral delay model of single-species population growth," Journal of Mathematical Analysis and Applications, vol. 259, no. 1, pp. 8-17, 2001.

[14] Z. H. Yang and J. D. Cao, "Sufficient conditions for the existence of positive periodic solutions of a class of neutral delay models," Applied Mathematics and Computation, vol. 142, no. 1, pp. 123142, 2003.

[15] S. P. Lu, "On the existence of positive periodic solutions for neutral functional differential equation with multiple deviating arguments," Journal of Mathematical Analysis and Applications, vol. 280, no. 2, pp. 321-333, 2003.

[16] Z. H. Yang and J. D. Cao, "Existence of periodic solutions in neutral state-dependent delays equations and models," Journal of Computational and Applied Mathematics, vol. 174, no. 1, pp. 179-199, 2005.

[17] Y. K. Li and Y. Kuang, "Periodic solutions of periodic delay Lotka-Volterra equations and systems," Journal of Mathematical Analysis and Applications, vol. 255, no. 1, pp. 260-280, 2001.

[18] R. E. Gaines and J. L. Mawhin, Coincidence Degree, and Nonlinear Differential Equations, Springer, Berlin, Germany, 1977.

[19] N. P. Các and J. A. Gatica, "Fixed point theorems for mappings in ordered Banach spaces," Journal of Mathematical Analysis and Applications, vol. 71, no. 2, pp. 547-557, 1979.

[20] D. J. Guo, "Positive solutions to nonlinear operator equations and their applications to nonlinear integral equations," Advances in Mathematics, vol. 13, no. 4, pp. 294-310, 1984. 


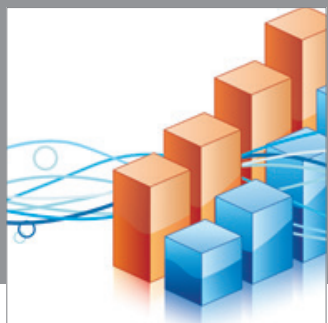

Advances in

Operations Research

mansans

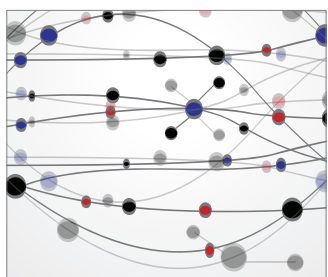

The Scientific World Journal
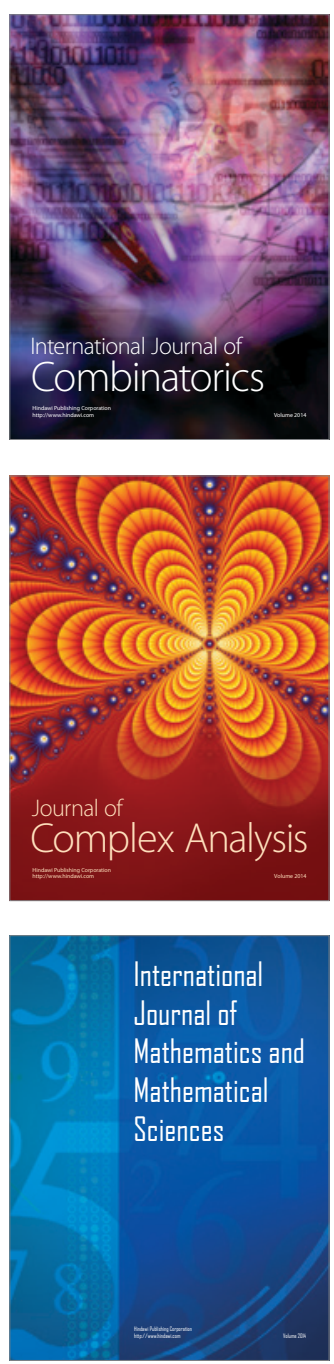
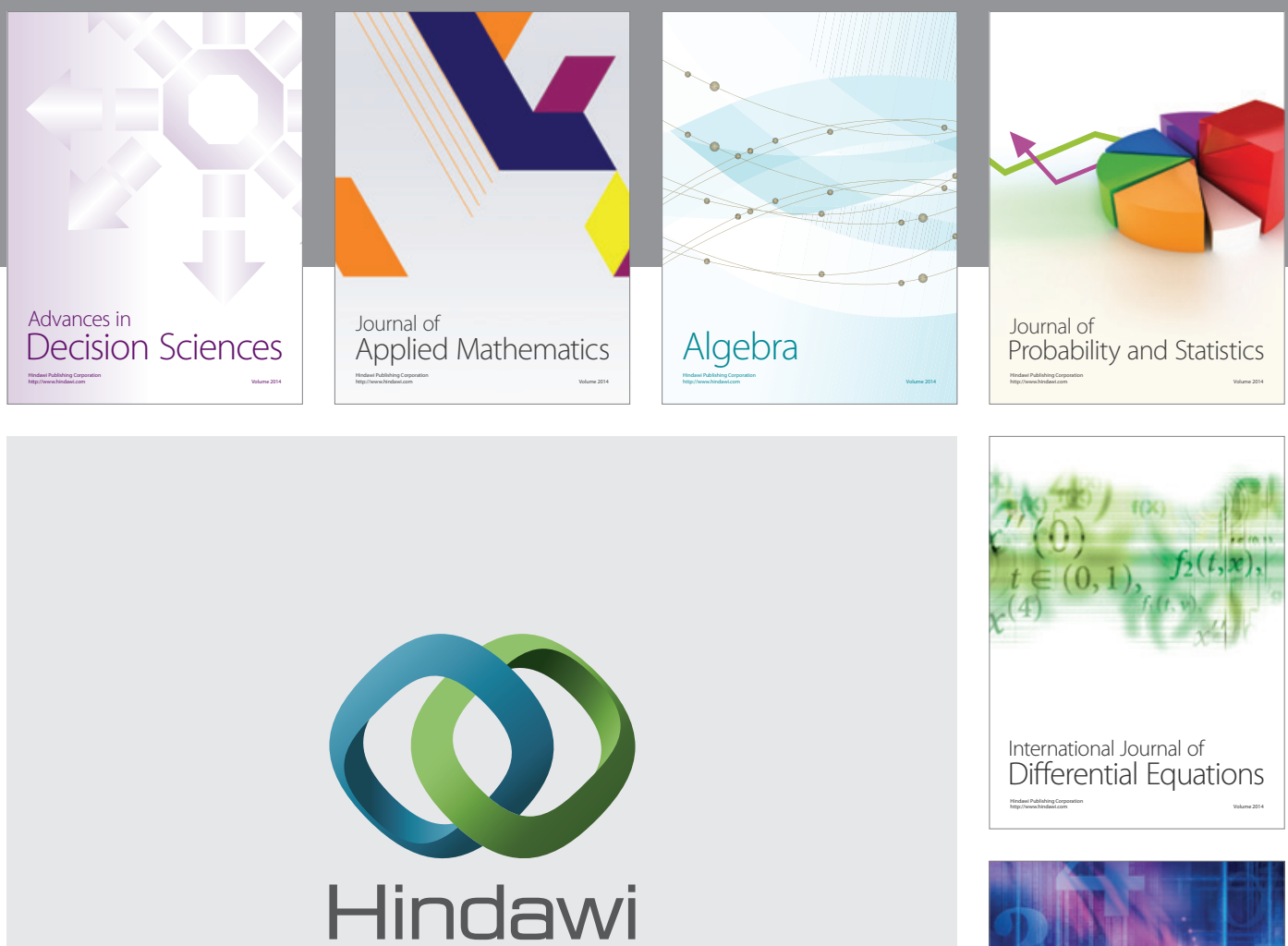

Submit your manuscripts at http://www.hindawi.com
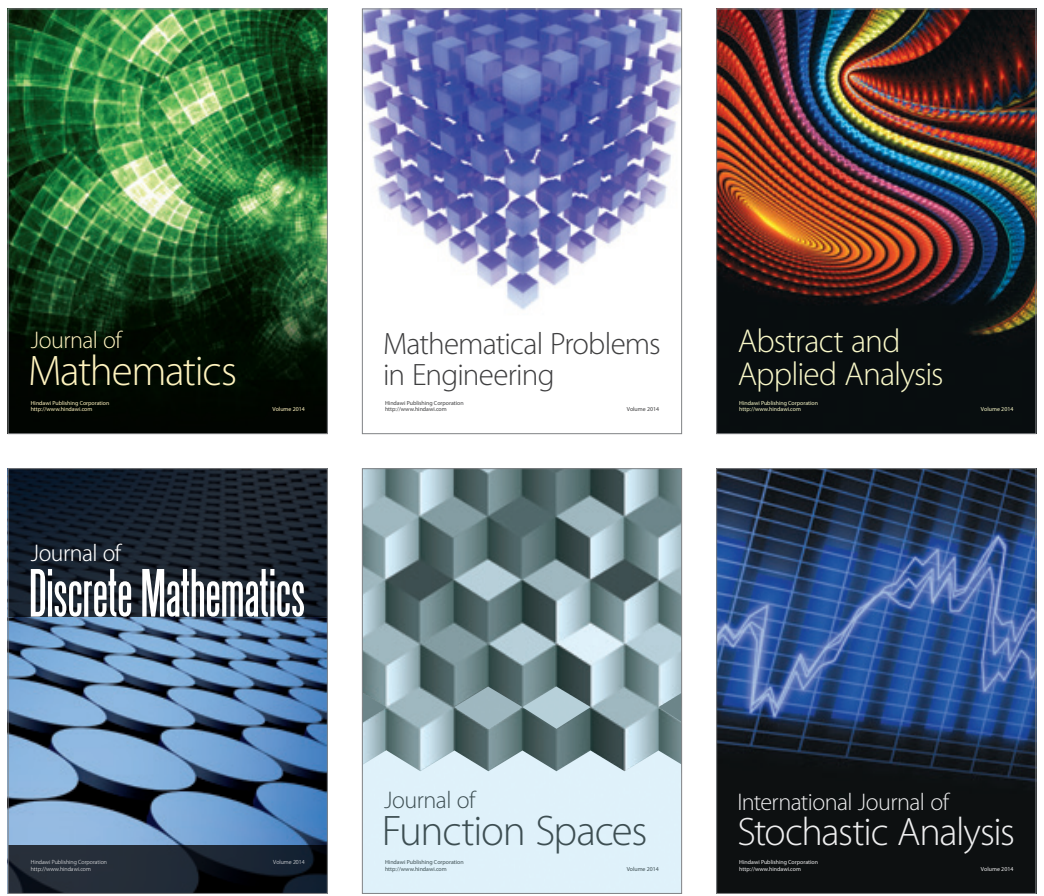

Journal of

Function Spaces

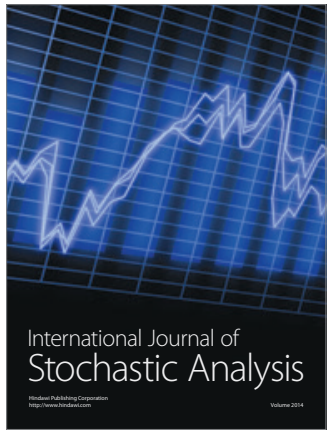

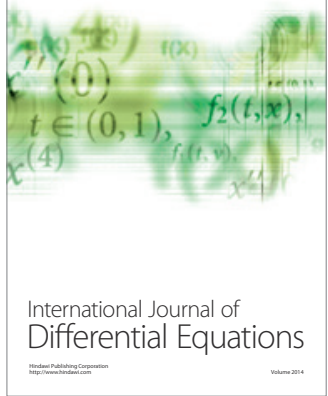
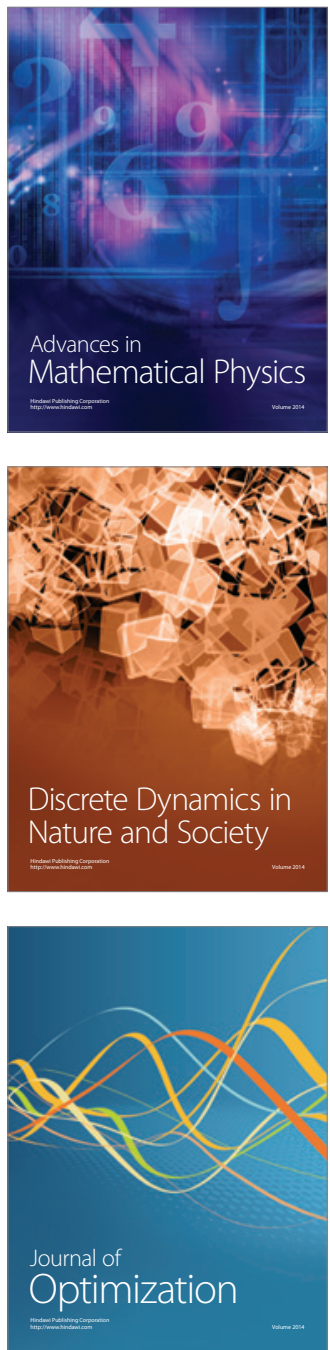